\title{
High-efficiency resonant rf spin rotator with broad phase space acceptance for pulsed polarized cold neutron beams
}

\author{
P.-N. Seo, ${ }^{1, *}$ L. Barrón-Palos, ${ }^{2, \dagger}$ J. D. Bowman, ${ }^{1, \$}$ T. E. Chupp,${ }^{3}$ C. Crawford, ${ }^{4, \S}$ M. Dabaghyan, ${ }^{5}$ M. Dawkins, ${ }^{6}$
} S. J. Freedman, ${ }^{7}$ T. Gentile, ${ }^{8}$ M. T. Gericke, ${ }^{9}$ R. C. Gillis, ${ }^{9, \|}$ G. L. Greene,${ }^{4,10}$ F. W. Hersman, ${ }^{5}$ G. L. Jones, ${ }^{11}$ M. Kandes, ${ }^{3}$ S. Lamoreaux, ${ }^{1, \mathbb{}}$ B. Lauss, ${ }^{7, * *}$ M. B. Leuschner, ${ }^{6, \dagger}$ R. Mahurin, ${ }^{4}$ M. Mason, ${ }^{5, *}$ J. Mei, ${ }^{6}$ G. S. Mitchell, ${ }^{1,8}$ H. Nann, ${ }^{6}$ S. A. Page, ${ }^{9}$ S. I. Penttilä, ${ }^{1, \dagger}$ W. D. Ramsay, ${ }^{9,12}$ A. Salas Bacci, ${ }^{1}$ S. Santra, ${ }^{6,\|\| l}$ M. Sharma, ${ }^{3}$ T. B. Smith, ${ }^{13}$ W. M. Snow, ${ }^{6}$ W. S. Wilburn, ${ }^{1}$ and H. Zhu, ${ }^{5, \pi I I}$

${ }^{1}$ Los Alamos National Laboratory, Los Alamos, New Mexico 87545, USA

${ }^{2}$ Arizona State University, Tempe, Arizona 85287, USA

${ }^{3}$ University of Michigan, Ann Arbor, Michigan 48104, USA

${ }^{4}$ Department of Physics University of Tennessee, Knoxville, Tennessee 37996, USA

${ }^{5}$ Department of Physics, University of New Hampshire, Durham, New Hampshire 03824, USA

${ }^{6}$ Department of Physics, Indiana University, Bloomington, Indiana 47405, USA

${ }^{7}$ Department of Physics, University of California, Berkeley, California 94720, USA

${ }^{8}$ National Institute of Standards and Technology, Gaithersburg, Maryland 20899, USA

${ }^{9}$ Department of Physics, University of Manitoba, Winnipeg, Manitoba, Canada R3T2N2

${ }^{10}$ Oak Ridge National Laboratory, Oak Ridge, Tennessee 37831, USA

${ }^{11}$ Department of Physics, Hamilton College, Clinton, New York 13323, USA

${ }^{12}$ TRIUMF, 4004 Wesbrook Mall, Vancouver, British Columbia, Canada V6T2A3

${ }^{13}$ Department of Physics, University of Dayton, Dayton, Ohio 45469, USA

(Received 4 October 2007; published 6 August 2008)

High precision fundamental neutron physics experiments have been proposed for the intense pulsed spallation neutron beams at JSNS, LANSCE, and SNS to test the standard model and search for new physics. Certain systematic effects in some of these experiments have to be controlled at the few ppb level. The NPDGamma experiment, a search for the small parity-violating $\gamma$-ray asymmetry $A_{\gamma}$ in polarized cold neutron capture on parahydrogen, is one example. For the NPDGamma experiment we developed a radio-frequency resonant spin rotator to reverse the neutron polarization in a $9.5 \mathrm{~cm} \times 9.5 \mathrm{~cm}$ pulsed cold neutron beam with high efficiency over a broad cold neutron energy range. The effect of the spin reversal by the rotator on the neutron beam phase space is compared qualitatively to rf neutron spin flippers based on adiabatic fast passage. We discuss the design of the spin rotator and describe two types of transmission-based neutron spin-flip efficiency measurements where the neutron beam was both polarized and analyzed by optically polarized ${ }^{3} \mathrm{He}$ neutron spin filters. The efficiency of the spin rotator was measured at LANSCE to be $98.8 \pm 0.5 \%$ for neutron energies from 3 to $20 \mathrm{meV}$ over the full phase space of the beam. Systematic effects that the rf spin rotator introduces to the NPDGamma experiment are considered.

DOI: 10.1103/PhysRevSTAB.11.084701

PACS numbers: 29.27.Hj, 85.75.- d, 25.40.Lw

\footnotetext{
*Corresponding author.

Triangle Universities Nuclear Laboratory, Durham, NC 27708, USA.

pilneyo@tunl.duke.edu

${ }^{\dagger}$ Present address: Universidad Nacional Autónoma de México, México, D.F. 04510, México.

${ }^{\ddagger}$ Present address: Oak Ridge National Laboratory, Oak Ridge, TN 37831, USA.

${ }^{\S}$ Present address: University of Kentucky, KY 40506, USA.

"Present address: Department of Physics, Indiana University, Bloomington, IN 47405, USA.

"Present address: Department of Physics, Yale University, New Haven, CT 06520, USA.

**Present address: Paul Scherrer Institut, CH-5232 Villigen, Switzerland.

${ }^{\dagger \dagger}$ Present address: Procure, Bloomington, IN 47408, USA.

\$Present address: Texas A\&M University, College Station, TX 77843, USA.

${ }^{\S}$ Present address: Department of Biomedical Engineering, University of California, Davis, CA 95616, USA.

"III Present address: Bhabha Atomic Research Center, Trombay, Mumbai 400085, India.

${ }^{\text {IIIT }}$ Present address: Canberra, CT, USA.
} 


\section{INTRODUCTION}

Reversal of the neutron polarization direction in sensitive nuclear, particle, and condensed matter physics experiments using polarized cold neutron beams is a simple method to control systematic uncertainty. Devices used for the spin reversal, usually called spin flippers, should ideally possess high spin-flip efficiency, operate reliably and efficiently over a broad distribution of neutron beam phase space, occupy a compact space, should not introduce any material into the neutron beam, and maintain compatibility with different types of neutron polarization devices present in experiments. As the continued increase in the brightness of pulsed spallation neutron sources makes polarized neutron measurements possible with a new level of statistical accuracy, there is also a need to improve and develop neutron spin flippers to accommodate the demands of these new types of experiments by taking advantage of the pulsed nature of the neutron source where time-offlight measurement is used to determine the neutron energy, to do so over an increased beam phase space, and to control possible sources of systematic uncertainties.

In this paper we describe the design and detailed characterization of a neutron spin flipper which we term a spin rotator. It is a resonant device based on performing NMR on a polarized neutron beam which moves through an orthogonal combination of static and rf magnetic fields in no DC field gradient. It is an active device in the sense that the amplitude of the rf magnetic field is varied in phase with the pulsed neutron source in such a way that the condition for rotating the neutron spins by $\pi$ radians in the rotator is always met for each subsequent neutron velocity class as it reaches the device. Such a resonant $\mathrm{rf}$ spin rotator for neutrons is by no means new: it has been implemented by many groups in the past [1]. What is new in our device is the combination of the breadth of neutron phase space over which the efficiency of the rotator is high, the accuracy and detail of the spin-flip efficiency measurements, and the variety of independent methods used to determine the efficiency including both the use of optically pumped polarized ${ }^{3} \mathrm{He}$ neutron spin filters and also the parity-violating $\gamma$-ray asymmetry on ${ }^{35} \mathrm{Cl}$. To achieve the required breadth of polarized neutron phase space coverage, we used neutron spin filters based on transmission through polarized ${ }^{3} \mathrm{He}$ gas in a new way, both as polarizers and as polarization analyzers. The ${ }^{3} \mathrm{He}$ technology is well suited to neutron polarimetry since the use of the ${ }^{3} \mathrm{He}$ spin filters allows the accurate determination of the beam polarization through relative neutron transmission measurements. In this work we have for the first time in neutron polarimetry measurements exploited another feature of polarized ${ }^{3} \mathrm{He}$ : namely, the possibility to use adiabatic fast passage (AFP) to reverse the ${ }^{3} \mathrm{He}$ polarization in order to determine the spin-flip efficiency. The comparison of the polarimetry measurement with and without the AFP constitutes a useful internal check on the measurements. Taken together, these achievements represent a qualitative advance in the art of neutron polarimetry and polarized neutron beam phase space manipulation.

\section{PRINCIPLES OF OPERATION OF RF NEUTRON SPIN FLIPPERS}

Neutron spin flippers based on rf magnetic fields are commonly used in experiments with polarized slow neutrons. There are two main types of rf flippers: adiabatic spin flippers and resonant spin rotators. The operating principle of both devices is well known from NMR [2] with small modifications due to the fact that the neutron spins are moving in the lab frame.

An adiabatic rf neutron spin flipper consists of a static magnetic field $B_{0}$, that changes in magnitude along a length of beam, and a perpendicular rf magnetic field whose magnitude varies with a maximum in the middle of the flipper. The operation of the flipper is best understood in the rotating frame of the neutron spin. If we choose the reference frame rotating at the Larmor frequency set by the static part of the field in the middle of the flipper and the $r f$ frequency to match this Larmor frequency, then in the rotating frame the transverse static field disappears and the longitudinal rf field becomes static. In this frame the neutron spin precesses about the remaining effective magnetic field which, as seen by the neutron, slowly varies from transverse up as the beam enters the flipper to longitudinal in the middle of the flipper and finally transverse down at the exit of the flipper. As long as the precession frequency of the neutron in this rotating frame about this slowly varying effective field is fast compared to the rate of change of the direction of the effective field, then the projection of the neutron spin onto the effective field is an adiabatic invariant and the spin follows the effective field with high accuracy. The probability of the depolarization during rotation depends exponentially on the adiabaticity parameter $\lambda=\left(\mu_{n} \frac{B_{0}{ }^{2}}{\hbar}\right) /\left(v \frac{\mathrm{d} B_{0}}{\mathrm{~d} z}\right)$, where $\mu_{n}$ is the neutron magnetic moment and $v$ the neutron velocity. The condition of the adiabatic rotation is easy to meet at slow neutron energies and such a spin flipper is relatively easy to realize. Adiabatic rf spin flippers for neutrons were first used for ultracold neutrons [3,4] and later also for cold neutron beams $[5,6]$. The theory for their operation has been further developed to take into account higher-order effects from Berry's phase $[7,8]$. The spin-flip efficiency can be close to unity for a broad spectrum of neutron energies as long as the neutrons satisfy the adiabaticity condition.

Resonant rf spin flippers also employ NMR principles. In this case, the static field is constant and the rf frequency is chosen to match the Larmor precession frequency throughout some region of space. When the neutron with speed $v$ passes through this region and meets the resonance condition, its spin is rotated by an angle of $\pi$ radians. 
Unlike the adiabatic spin flipper, the spin-flip efficiency of the resonant spin rotator is directly sensitive to the neutron velocity. It therefore possesses high efficiency only for neutrons in a narrow class of velocities.

However, the action of these two types of spin flippers on the phase space of the beam is not quite the same. Since the adiabatic flipper by necessity possesses a static magnetic field gradient, the Stern-Gerlach effect $\left(\vec{F}=-\mu_{n}\right.$. $\left.\vec{\nabla} B_{0}\right)$ in such a flipper will steer the neutron beam in slightly different directions for the two spin states. In addition, by Maxwell's equations the static magnetic field gradient necessarily leads to field gradients in regions outside of the spin flipper. Finally, since the static magnetic field is different at the two ends of the device, the neutron kinetic energy can change. As we discuss below, a resonant rf spin flipper need possess none of these effects.

An experiment which places stringent limits on neutron spin-dependent phase space changes is the NPDGamma experiment, a measurement of the small parity-violating weak $\pi$-nucleon coupling constant, $f_{\pi}^{1}$, in the reaction of $\vec{n} \rightarrow p \rightarrow d+\gamma$. In the experiment polarized cold neutrons are captured by protons in a liquid parahydrogen target and the $\gamma$-ray asymmetry $A_{\gamma}$ with respect to the neutron beam polarization is measured [9]. The experiment determines $f_{\pi}^{1}$ unambiguously by measuring $A_{\gamma}$ with the precision of $1 \times 10^{-8}$ which is $20 \%$ of its predicted value [10].

At this level of precision, spin-dependent changes in the phase space of the polarized neutron beam must be strictly minimized. The Stern-Gerlach effect that would necessarily be present in an adiabatic rf spin flipper will deflect the neutron beam along the neutron polarization direction. The resulting spin-correlated spatial shift in the neutron capture distribution in the hydrogen target will change the solid angle seen by the $\gamma$ detectors, and would result in an asymmetry in the signal of the $\gamma$-detector array that is difficult to distinguish from the parity-violating signal that the experiment measures. Although this effect can be minimized by employing a smaller static field gradient in the adiabatic flipper, this requires the length of the flipper to be increased to maintain the adiabatic condition for all of the neutrons in the spectrum and therefore requires all downstream elements of the apparatus to be widened to continue to accept all events from the diverging cold neutron beam. To eliminate this potential source of the systematic uncertainty, we decided to design a rf spin rotator that possesses negligible static field gradients.

We call this device a rf spin rotator (RFSR) as opposed to a rf spin flipper to emphasize that this device does not change the kinetic energy of the neutrons on resonance. The change in potential energy of the neutron inside the rf field region is caused by the exchange of energy of the neutron with the rf field leaving the kinetic energy unchanged. There are two equivalent ways to see why this spin rotator has this property.

\section{A. Semiclassical view}

Consider a monoenergetic neutron wave packet moving along the $+\hat{y}$ axis starting from $-\infty$ with an initial polarization in the $+\hat{z}$ direction. Let it move in a uniform and static magnetic field $B_{0} \hat{z}$, and in a uniform and timedependent magnetic field which rotates normal to the $\hat{z}$ axis with frequency $\Omega, \vec{B}(t)=\hat{y} B_{1} \cos (\Omega t)+\hat{x} B_{1} \sin (\Omega t)$, over a length $d$ on the $+\hat{y}$ axis. The magnitude of the static field is given by $\hbar \omega_{0}=\mu_{n} B_{0}$ and the Larmor precession frequency is $\omega_{L}=2 \mu_{n} B_{0} / \hbar$. Differences between this model and the real spin rotator are small.

Consider the semiclassical view used in NMR in which one performs a transformation from the inertial rest frame of the neutron into a frame rotating about the $+\hat{z}$ axis with frequency $\Omega$. If $\Omega=\omega_{\mathrm{L}}$ (on resonance), then in the rotating reference frame and in the rf field region the neutron sees only an effective magnetic field $\vec{B}_{\text {eff }}=B_{1} \hat{y}$ along the $+\hat{y}$ axis which is normal to the initial spin direction. Therefore, in this frame the neutron starts to precess about the $+\hat{y}$ axis and if the time it spends in the rf field is just right then the spin rotates by $\pi$ radians before exiting the region. Notice that if we also view the motion of the neutron before and after the rf field region in this rotating frame then the neutron never experiences a change in the effective field along the $+\hat{z}$ axis. But this change is what would be required to change the potential energy of the neutron. In addition, the transformation to the rotating frame removes the energy exchange of the neutron with the rf field by turning this into a static field. Therefore $\vec{\mu}_{n}$. $\vec{B}_{\text {eff }}=0$ in the rotating frame on resonance and the kinetic energy of the neutron does not change.

This is not true off resonance where $\Omega \neq \omega_{L}$ and the effective magnetic field possesses a component along the $+\hat{z}$ axis when the neutron enters the rf field region. There is a change in potential energy at boundary and, therefore, a change in the neutron kinetic energy. In the rotating frame the effective field makes an angle $\tan \theta=\left(\omega_{L}-\Omega\right) / \omega_{1}$, where $\hbar \omega_{1}=\mu_{n} B_{1}$, and if the neutron spends the same amount of time in the rf field then it precesses to an angle $\pi /(2-2 \theta)$ and a change in its potential energy, as it transverses the boundary of the rf region, is $\hbar \Omega \cos \pi /(2-$ $2 \theta) \approx \hbar\left(\omega_{L}-\Omega\right)$ for small values of $\theta$.

\section{B. Quantum-mechanical view}

The same result is obtained using a quantum-mechanical approach. One can solve for the time-dependent wave functions for the spin-up and spin-down components by the methods of one-dimensional quantum mechanics $[11,12]$. The derivation assumes a wave function of the form for a plane wave traveling in the $+\hat{y}$ direction

$$
\psi^{ \pm}(y, t)=\left[\begin{array}{l}
A_{n}{ }^{ \pm} \\
B_{n}{ }^{ \pm}
\end{array}\right] e^{i k_{n} y} e^{-i\left(\omega_{n} \pm \Omega / 2\right) t} .
$$

Here $n$ indexes the three regions of space; before, inside, 
and after the time-dependent field region located in $0 \leq$ $y \leq d$, and \pm refers to up/down spin states. Then one matches the wave functions and their derivatives in the usual way at two boundaries. This procedure generates 16 different components to the time-dependent wave function in the rf field region. For each of the two spin states and for each of the reflected and transmitted waves, there are four independent components of the wave function. Two components correspond to the two different momenta in the dispersion relation generated by substituting $\psi^{ \pm}(y, t)$ into the Schrödinger equation and for the case of no energy exchange with the time-dependent field. In the region before the rf field these momenta are $k_{i}^{+}=\sqrt{\omega_{\text {kin }}-\overline{\omega_{0}}}$ for spin up and $k_{i}^{-}=\sqrt{\omega_{\text {kin }}+\omega_{0}}$ for spin down, $k_{i}=$ $\sqrt{\omega_{\text {kin }}}$ represents the wave number ( $\omega_{\text {kin }}$ kinetic energy) of the neutron in vacuum; no static or rf fields are present. To simplify derivation, units $\hbar=2 m=1$, where $m$ is the neutron mass, are used. The other two components correspond to these two momenta with energy exchange with the rf field. Under the usual assumptions that the timedependent magnetic field is weak enough that only one $\mathrm{rf}$ photon is exchanged between the neutron and the rf field, and that the kinetic energy is large compared to the potential energy of the magnetic moment in the fields and also to the energy exchanged with the time-dependent field, one can solve for the amplitudes for the neutron spin to be up or down in the region past the time-dependent field $y \geq d$ assuming it entered with spin up. Using the notation of Ref. [11], the transmitted wave function becomes

$$
\zeta^{ \pm}(y, t)=\left[\begin{array}{l}
T_{0}^{+} e^{-i \omega_{0} y / v} \\
T_{1}^{-} e^{-i\left(\Omega-\omega_{0}\right) y / v} e^{i \Omega t}
\end{array}\right] e^{i\left(k_{i} y-\omega_{\text {kin }} t\right)}
$$

with $T_{0}^{+}=e^{-i \varepsilon d / v}\left[\cos \left(\omega_{\mathrm{A}} d / v\right)+i\left(\varepsilon / \omega_{\mathrm{A}}\right) \sin \left(\omega_{\mathrm{A}} d / v\right)\right]$ and $T_{1}^{-}=-i\left(\omega_{1} / \omega_{\mathrm{A}}\right) e^{i \varepsilon d / v} \sin \left(\omega_{\mathrm{A}} d / v\right)$. Here $\omega_{\mathrm{A}}^{2}=$ $\omega_{1}^{2}+\varepsilon^{2}, \varepsilon=\Omega / 2-\omega_{0}$, and $\omega_{1} \ll \omega_{0}$. Then, the spinflip probability is given by

$$
f=\left|T_{1}^{-}\right|^{2}=\frac{\omega_{1}^{2}}{\omega_{\mathrm{A}}^{2}} \sin ^{2}\left(\omega_{\mathrm{A}} d / v\right) .
$$

On resonance $\varepsilon=0$ and for $\omega_{1} d / v=\pi / 2, f$ is unity. For the final spin-down state we can read off the momentum and energy from the wave function: $k^{-}=k_{i}^{+}-\omega_{0} / v$ and total energy $\omega=\omega_{\text {kin }}-2 \omega_{0}$. Neglecting quadratic term of the Zeeman energy, the kinetic energy is $k^{-2} \cong$ $k_{i}^{+2}-2 \omega_{0} k_{i}^{+} / v=\omega_{\text {kin }}-\omega_{0}$. Comparing with the initial kinetic energy of the spin-up neutron before entering the rf field, $k_{i}^{+2}=\omega_{\text {kin }}-\omega_{0}$, we see that indeed the kinetic energy of the neutron has not changed in agreement with the conclusion drawn using the rotating reference frame.

Off resonance the momentum and total energy are $k^{-}=$ $k_{i}^{+}-\left(2 \varepsilon+\omega_{0}\right) / v$ and $\omega^{-}=\omega_{\text {kin }}-\Omega$. The kinetic energy is $k^{-2} \cong k_{i}^{+2}-4 \varepsilon k_{i}^{+} / v-2 \omega_{0} k_{i}^{+} / v=\omega_{\text {kin }}-$ $\omega_{0}-2 \varepsilon$ and a change in kinetic energy is $-2 \varepsilon$ as determined above. The conclusion of this derivation is the same: on resonance where $\varepsilon=0$, the neutron kinetic energy is not changed by the interaction with the rf field.

How does the realistic RFSR device deviate from this idealized case? First, the rf field direction does not rotate in space as assumed but oscillates in magnitude. As usual, one can decompose the rf field as a sum of a rotating and a counter-rotating component, and if one is close to resonance then the other is far from resonance and is negligible for the small amplitudes of the rf fields employed in this work. Second, the amplitude of the rf field is in fact not uniform inside the spin rotator. Instead it has a spatial distribution along the trajectory of the neutron determined by the boundary conditions of the rf field inside the aluminum shield. However, it is clear that on resonance this nonuniformity still does not lead to a change in the kinetic energy of the neutrons to first order because the effective field, although now time dependent, still satisfies $\vec{\mu}_{n}$. $\vec{B}_{\text {eff }}=0$ in the rotating reference frame. Therefore even a realistic rf spin rotator will leave the kinetic energy of the neutron beam unchanged to high accuracy.

\section{DESCRIPTION OF THE APPARATUS}

Figure 1 shows a schematic of the NPDGamma experiment and setup for the measurement of the spin-flip efficiency in the pulsed cold neutron beam line flight path 12 (1FP12) at the Los Alamos Neutron Science Center (LANSCE). Neutrons from the $20 \mathrm{~Hz}$ spallation target are moderated by the 1FP12 partially coupled cold hydrogen moderator [13] and delivered by a $20.7 \mathrm{~m}$ long supermirror $(m=3)$ neutron guide with cross sectional area of $9.5 \mathrm{~cm} \times 9.5 \mathrm{~cm}$ to a magnetic and radiological shielding structure which houses the experiment. A $m=3$ supermirror neutron guide transmits the neutrons with transverse velocities $<20 \mathrm{~m} / \mathrm{sec}$ with high efficiency. To prevent frame overlap of the neutrons from different pulses which would degrade the one-to-one correlation of neutron energy with a time-of-flight, the neutrons with energy less than $1 \mathrm{meV}$ are absorbed by a frame-definition chopper located between the moderator and the experiment [14].

Neutron beam monitors M1, M2, and M3 are parallel plate ${ }^{3} \mathrm{He}$ ionization chambers filled with an admixture of ${ }^{3} \mathrm{He},{ }^{4} \mathrm{He}$, and $\mathrm{N}_{2}$ to a total pressure of one atmosphere [15]. The stable and linear neutron beam monitor M1 is used to normalize the neutron beam intensity pulse by pulse with $1 \%$ accuracy. Monitors M1 and M2 are used for the beam polarization measurement. A monitor pair M2 and $\mathrm{M} 3$ is used to follow the ortho-para ratio of the liquid parahydrogen target of the NPDGamma experiment (the target is not shown in Fig. 1) [16], to study the depolarization of the neutron beam in the hydrogen target, and to measure the spin-flip efficiency of the rf spin rotator. The monitors M1 and M2 each absorb 4\% of the beam at $4 \mathrm{meV}$ and the M3 absorbs all the incident cold neutrons.

For the spin-flip efficiency measurement, the beam is collimated to $10 \mathrm{~cm}$ diameter using ${ }^{6} \mathrm{Li}$-loaded epoxy 


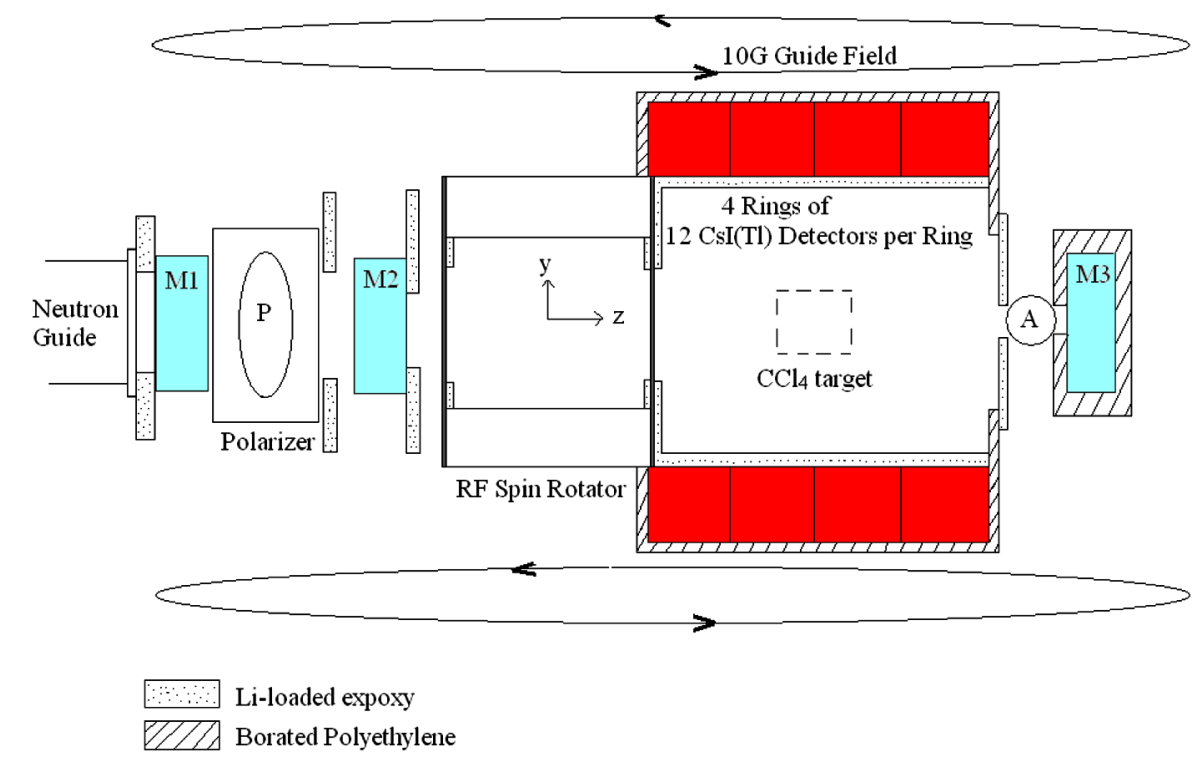

FIG. 1. (Color) A schematic side view (not to scale) of the NPDGamma apparatus and setup for the spin-flip efficiency measurement on 1FP12 at LANSCE. M1, M2, and M3 are ${ }^{3} \mathrm{He}$ ion chambers. The polarized ${ }^{3} \mathrm{He}$ spin filter cell P is placed between M1 and M2 and the polarized ${ }^{3} \mathrm{He}$ analyzer cell $\mathrm{A}$ is mounted in the front of $\mathrm{M} 3$ which is $1.65 \mathrm{~m}$ downstream from the exit window of the neutron guide. The analyzer cell and M3 are used for a measurement of the spin-flip efficiency and to monitor the polarization of the beam passing through the liquid parahydrogen target (not shown in the figure). Beam collimators are made from ${ }^{6} \mathrm{Li}$-loaded epoxy material and neutron shielding from borated polyethylene. The entire apparatus is in a uniform $1 \mathrm{mT}$ magnetic field.

material ( $9 \%$ by weight, $90 \%$ enrichment of $\left.{ }^{6} \mathrm{Li}\right)$. After M1 the unpolarized neutrons enter the spin filter, a cell of polarized ${ }^{3} \mathrm{He}$ gas [17]. Because of the large spindependent neutron- ${ }^{3} \mathrm{He}$ capture cross section, neutrons with spin parallel to the ${ }^{3} \mathrm{He}$ spin, the triplet spin state, are transmitted without significant attenuation, while those with antiparallel spin are absorbed. The ${ }^{3} \mathrm{He}$ cell of $12 \mathrm{~cm}$ in diameter can polarize the entire cold neutron beam phase space. The magnitude of the polarization variation with neutron transverse momentum is less than $0.1 \%$. The spin filter is operated in a $1 \mathrm{mT}$ homogeneous $\left(\Delta B_{0} / \Delta r<\right.$ $10 \mu \mathrm{T} / \mathrm{m})$ vertical magnetic field. The polarized beam passes through a second $10 \mathrm{~cm}$ collimator before entering M2. For the measurement, a third $10 \mathrm{~cm}$ diameter collimator is inserted on the downstream side of M2. The neutron beam polarization is calculated from relative neutron transmission intensities of the monitors M1 and M2 [17,18].

A resonant RFSR is used for neutron spin reversal at $20 \mathrm{~Hz}$. In the NPDGamma experiment after the RFSR, the polarized cold neutrons enter a liquid parahydrogen target $\left(\mathrm{LH}_{2}\right)$, where they are captured by protons. Deuterons from the capture reaction decay to the ground state by emitting $2.2-\mathrm{MeV} \gamma$-rays that are detected by an array of $48 \mathrm{CsI}(\mathrm{Tl})$ detectors [19] viewed by vacuum photodiodes coupled to low-noise solid-state electronics [20]. The neutron polarization analyzer, a polarized ${ }^{3} \mathrm{He}$ cell in front of M3, is used to analyze the polarization of the beam that passes through the hydrogen target or the performance of the RFSR. Because of the small size of the spherical analyzer cell, $2.5 \mathrm{~cm}$ in diameter, the beam entering the analyzer is collimated to $1.3 \mathrm{~cm}$ diameter. M3 is shielded by borated polyethylene against background neutrons.

The neutron kinetic energy will change as discussed above if the static magnetic field changes. To minimize this effect and provide conditions for the resonant $\mathrm{rf}$ spin reversal, the entire experiment is immersed in a $1 \mathrm{mT}$ magnetic field. The homogeneity requirement of the field in the beam region is set by the need to suppress a possible source of a false asymmetry caused by the up-down SternGerlach steering of the polarized beam after the RFSR. An additional requirement for the field is that the direction of the field with respect to the up-down direction of the $\gamma$ detector in the location of the $\mathrm{LH}_{2}$ target has to be known with the accuracy of $20 \mathrm{mrad}$ or better to reduce the size of the projection of possible parity-conserving left-right asymmetries along the vertical detector axis. The initial alignment was accomplished by turning the static field $\vec{B}_{0}$ parallel with the $\hat{y}$ axis by shim coils within the angular accuracy of a commercial three-axis flux-gate magnetometer and aligning the detector array with the same $\hat{y}$ axis using an optical survey instrument. The final up-down alignment of the detector was then performed with the neutron beam; a small Gd sample was placed to the middle of the detector and $\gamma$ rays from neutron capture on Gd were used to define effective locations of the $48 \mathrm{CsI}(\mathrm{Tl})$ detectors which were then used in the data analysis.

In the NPDGamma experiment, the asymmetry $A_{\gamma}$ is determined from $\gamma$-ray yields obtained by the upper half and the lower half of the $\gamma$-detector array for every neutron pulse. The $\gamma$ asymmetry in 1FP12 is measured with the 
accuracy of about $10^{-3}$ per neutron pulse with $100-\mu \mathrm{A}$ average proton current incident on the spallation target. Since it is not practical to require the efficiencies of the detector halves to be equal to this precision, the spin rotator is needed to reverse the sign of the asymmetry with respect to the $\gamma$-detector array.

The main handle to control systematic uncertainties in the NPDGamma experiment is therefore the frequent reversal of the neutron spin direction. The neutron polarization can be reversed with two independent spin flips without changing the static magnetic field. The polarization direction of the ${ }^{3} \mathrm{He}$ in the spin filter can be reversed with the adiabatic fast passage (AFP) technique. The frequent use of this spin flip is limited by the time of a few seconds it takes to perform the flip and a slow recovery of a small ${ }^{3} \mathrm{He}$ polarization loss per AFP. The RFSR was designed to reverse the neutron spin direction at frequencies from zero to $60 \mathrm{~Hz}$ and was chosen to match the $20 \mathrm{~Hz}$ pulse frequency of the LANSCE spallation source. Although a third neutron spin flip can in principle be performed by reversing the direction of the vertical $1 \mathrm{mT}$ static field, a potential slow relaxation of the magnetization in the steel of the magnetic shielding precluded the use of this method at 1FP12.

In the LANSCE spallation source neutrons are produced by short (a few hundred nanoseconds long) proton pulses which strike a tungsten target. The moderated neutrons have an energy distribution and therefore they have different arrival times in the RFSR.

Before entering the RFSR, the neutron spin precesses in the static field of $\vec{B}_{0} \hat{y}$ where $\left|\vec{B}_{0}\right|=1 \mathrm{mT}$; see Fig. 1 for the coordinates. Inside the RFSR the static and time-dependent magnetic fields are present and in the rotating reference frame the spin rotates with angular frequency of $\omega_{1}=\frac{\mu_{n} B_{1}}{\hbar}$ about the effective magnetic field $\vec{B}_{\text {eff }}=\left(B_{0}-\frac{\Omega}{2 \mu_{n}} \hbar\right) \hat{y}+$ $B_{1} \hat{z}$ which on resonance is $\vec{B}_{\text {eff }}=B_{1} \hat{z}$ and the neutron spin rotates in the $\hat{x} \hat{y}$ plane. To rotate the incoming spin by $\pi$ radians, the spin has to remain in the rf field for a time $t=n \pi \hbar / \mu_{n} B_{1}$, where $n=1,3,5, \ldots$. Since the length $L$ of the spin rotator is fixed, the time depends on neutron velocity $v$. If the time is $t=L / v$, then the condition for the $\pi$-radian rotation is satisfied. To achieve a high spin-flip efficiency for each neutron velocity, the amplitude $B_{1}$ has to be

$$
B_{1}=\frac{n \pi \hbar}{\mu_{n}}\left(\frac{l}{L}\right) \frac{1}{t_{\mathrm{tof}}}
$$

where $l$ is the distance from the moderator to the spin rotator (22 $\mathrm{m}$ in the NPDGamma experiment on 1FP12) and $t_{\text {tof }}$ is the neutron time of flight. A result of Eq. (3.1) is that the rf field strength $B_{1}$ has to be varied during a neutron pulse as $1 / t_{\text {tof }}$.

According to Eq. (2.1) the spin-flip probability for a neutron with velocity $v$ depends upon $B_{1}$ and $\left(\omega_{0}-\right.$ $\Omega / 2$ ), since time $t$ that the neutron spends in the RFSR is

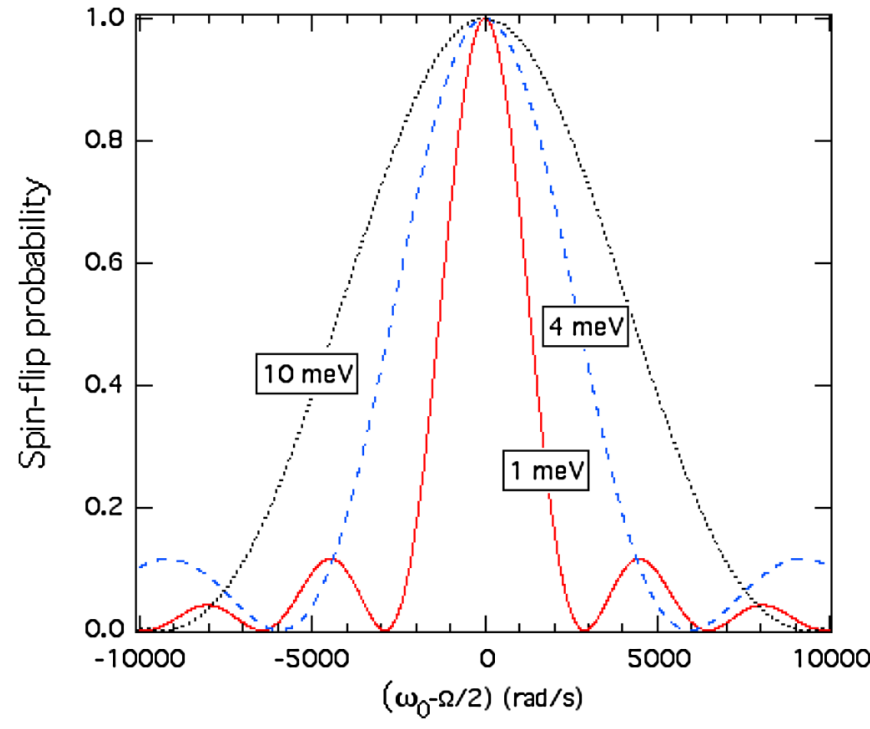

FIG. 2. (Color) Spin-flip probability as a function of $\left(\omega_{0}-\right.$ $\Omega / 2$ ) for the RFSR of the NPDGamma experiment at three neutron energies, see Eq. (2.1). At each energy the amplitude of $\mathrm{rf}$ field $B_{1}$ has been selected so that the spin-flip probability is unity on resonance.

fixed. In the real RFSR $B_{1}$ is not constant on different neutron trajectories because the field is produced by a solenoid with a finite length. Therefore, a condition that $\Omega / 2 \neq \omega_{0}$ can be a consequence of an inhomogeneous $\mathrm{rf}$ magnetic field across the RFSR or of $\Omega$ is not perfectly tuned to resonance.

Figure 2 shows the spin-flip probability calculated using Eq. (2.1) for the $30 \mathrm{~cm}$ long RFSR solenoid as a function of $\left(\omega_{0}-\Omega / 2\right)$ for three different neutron energies; $1 \mathrm{meV}$ (solid line), $4 \mathrm{meV}$ (dashed line), and $10 \mathrm{meV}$ (dotted line). The rf amplitude $B_{1}$ is selected so that on resonance the neutron spin rotates by $\pi$ radians. In the $1 \mathrm{mT}$ static field the $10-\mathrm{meV}$ neutron performs eight precessions in the $\mathrm{rf}$ field whereas the spin of a $1 \mathrm{meV}$ neutron performs 30 precessions. A 4- $\mu \mathrm{T}$ change in the static field moves the spin out of resonance and reduces the spin-flip probability for $1 \mathrm{meV}$ neutron by $4.5 \%$, for $4 \mathrm{meV}$ neutron by $1.1 \%$, and for $10 \mathrm{meV}$ neutron by $0.5 \%$, respectively. A $10 \%$ change in the optimum strength of $B_{1}$ reduces the spin-flip probability by $2.5 \%$ on resonance.

\section{DESIGN AND CHARACTERIZATION OF THE RF RESONANT SPIN ROTATOR}

\section{A. The rf magnetic field design for the rf resonant spin rotator}

The rf field of the RFSR is produced by a solenoid inside an aluminum shield and can be driven at Larmor frequency of approximately $29 \mathrm{kHz}$ for the $1 \mathrm{mT}$ static field. The amplitude $B_{1}$ is not constant inside the spin rotator since it is produced by solenoid with a finite radius and it is modified by the boundary conditions from the aluminum 
shielding. A more appropriate design parameter is the integral of the amplitude of the rf field along the neutron trajectory over the length of the spin rotator,

$$
\int_{-L / 2}^{L / 2} B_{1}(r, z) \mathrm{d} z
$$

where $r$ is the radial distance from the axis. This integral is directly proportional to the number of precessions of the neutron polarization along the effective magnetic field in the rotating frame. In order to achieve high spin-flip efficiency, the neutron spin has to perform several Larmor precessions about the effective field in the rotating frame as it passes through the spin rotator.

The dimensions of the solenoid and its aluminum rf shielding enclosure have been optimized for the neutron beam phase space available on FP12 at LANSCE. The spin rotator can accept a $10 \mathrm{~cm} \times 10 \mathrm{~cm}$ beam area. The length of the solenoid is defined by the rf amplitude $B_{1}$ and the adiabatic requirement. These factors limit the maximum neutron energy which can be reversed by our RFSR to $\sim 100 \mathrm{meV}$. The diameter was optimized for high spin-flip efficiency resulting in a length-to-diameter ratio of 1:1. The aluminum shielding dimensions must be larger than the rf solenoid to reduce the transverse gradients at the position of the beam entrance and exit windows, which could lead to substantial depolarization of the neutrons on off-axis trajectories. The RFSR must also possess efficient electromagnetic shielding to prevent rf power from reaching the rest of the experiment and potentially causing a false systematic effect.

To satisfy these design criteria, we chose a rf solenoid of $S=15 \mathrm{~cm}$ in radius and $M=30 \mathrm{~cm}$ in length with its axis along the neutron beam. The solenoid has a single layer of 273 turns of 18 gauge $\mathrm{Cu}$ wire wound on a nylon spool. The rf field produced by the coil is attenuated by an Al cylinder $(R=20 \mathrm{~cm}, L=40 \mathrm{~cm})$ with $\mathrm{Al}$ caps at the ends. The thickness of the wall of this shielding is $5 \mathrm{~mm}$ and the beam entry and exit windows are $0.5 \mathrm{~mm}$ thick, which is the skin depth of the $29 \mathrm{kHz}$ rf field in aluminum. The entry and exit windows allow the neutron beam to pass the RFSR with less than $1 \%$ attenuation. Depolarization of the neutron beam in the aluminum windows is $<0.1 \%$.

\section{B. Control electronics for RFSR operation}

As described above, the amplitude of the rf magnetic field generated by the solenoid is matched to the velocity of the neutrons to rotate the spin direction by $\pi$ radians. Because the velocity of the neutrons is proportional to $1 / t_{\text {tof }}$, the amplitude $B_{1}$ must be changed as $1 / t_{\text {tof }}$ during each $50 \mathrm{~ms}$ long neutron pulse for $\pi$ radians rotation. For $100-\mathrm{meV}$ neutrons the required amplitude of the rf magnetic field is $0.38 \mathrm{mT}$, which is produced by a solenoid current of approximately $0.8 \mathrm{~A}$. To reduce the voltage requirement for the amplifier that drives the solenoid $(L=$ $8.3 \mathrm{mH})$, a capacitor $(C=3.6 \mathrm{pF})$ was added in series to produce a series resonant circuit with a resonant frequency approximately equal to the neutron spin precession frequency of $29 \mathrm{kHz}$.

Figure 3 shows the circuit used to drive the solenoid. A frequency generator (FG2) produces a $29 \mathrm{kHz}$ sine wave, which is amplified by a fixed-gain audio amplifier (A2). The output of the amplifier goes to a switchbox that selects whether the output is sent to the rotator coil or to a resistive dummy load, approximately equal to the impedance of the solenoid. The switch box also contains pickoff transformers to monitor current (X1) and voltage (X2) out of the amplifier. The outputs of these transformers are sampled at a rate of $62.5 \mathrm{kHz}$ by 16 bit analog-to-digital converters (ADCs), allowing reconstruction of the waveforms. Typical reconstructed current signals of the current sent to the solenoid (spin-down state) or to the dummy load (spin-up state) are shown in Fig. 4 left and right, respectively.

The amplitude of the $29 \mathrm{kHz}$ sine wave is varied to produce the required $1 / t_{\text {tof }}$ behavior by amplitude modulating FG2. This amplitude modulation is produced by comparing an ideal ramp signal generated by FG1 to a full-wave rectified output of X1 using difference amplifier A1 which includes a single-pole filter with a $3-\mathrm{dB}$ point of $1 \mathrm{kHz}$ to filter out the $29 \mathrm{kHz}$ component. This feedback mechanism maintains the accuracy of the amplitude ramp, compensating for gain and impedance drifts.

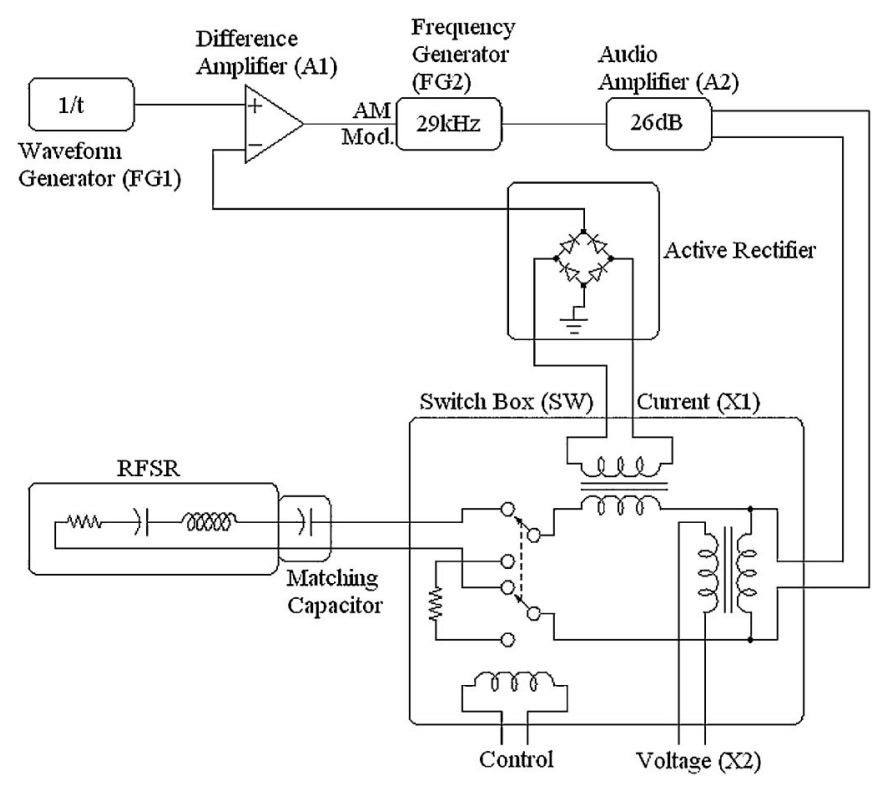

FIG. 3. Schematic of the control electronic circuit of the RFSR. An arbitrary waveform generator (FG1) sends a $1 / t$ ramp signal to a difference amplifier (A1). The amplifier outputs the signal difference between the $1 / t_{\text {tof }}$ signal and the rectified $29 \mathrm{kHz}$ feedback signal driven by the spin rotator coil. Current and voltage signals are measured by ADCs for each neutron pulse (see Fig. 4). 


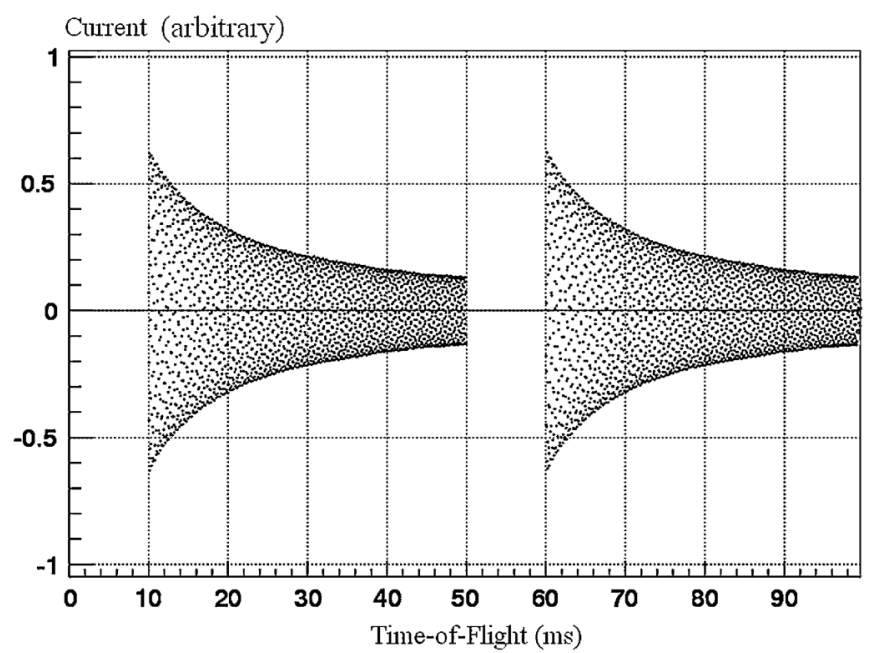

FIG. 4. Typical reconstructed current-to-voltage signals for the solenoid (left) and for the resistive dummy load (right) as measured by ADC. The signals were sampled at the rate of $62.6 \mathrm{kHz}$ over $40-\mathrm{ms}$ long neutron frame. Note that the signals start at $t_{\text {tof }}=0 \mathrm{~ms}$ but data acquisition starts at $10 \mathrm{~ms}$.

\section{Calculation of the rf fields}

The rf field was calculated from a magnetic scalar potential $B(r, z)=-\nabla \phi(r, z)$. The eddy currents in the aluminum cylinder were approximated by imposing the boundary conditions that the component of the magnetic field normal to the surface of the cylinder vanishes. Since the fields are cylindrically symmetric, the magnetic potential does not depend on the azimuthal angle.

Using the cylindrical coordinate system where the axis of symmetry is the $\hat{z}$ axis, variables were separated and then solutions for radial and axial components of the potential were sought in the form

$$
\begin{aligned}
\phi(r, z) & =f_{n}(r) g_{n}(z) \propto I_{0}\left(k_{n} r\right) \sin \left(k_{n} z\right) \\
& \propto K_{0}\left(k_{n} r\right) \cos \left(k_{n} z\right),
\end{aligned}
$$

where $I_{0}\left(k_{n} r\right)$ and $K_{0}\left(k_{n} r\right)$ are modified Bessel functions. For the field calculations, the volume of the aluminum cylinder was divided into two subvolumes; one inside (inner) and the other outside (outer) of the solenoid. Because the magnetic field is regular on the axis, at $r=$ 0 , the coefficient of the $K_{0}\left(k_{n} r\right)$ term vanishes. Hence, the coefficient of the $\cos \left(k_{n} r\right)$ term vanishes in both regions, the axial magnetic field must be an even function of $z$. With a boundary condition that at the ends of the cylinder $z=$ $\pm L / 2$ the $z$ component of the field vanishes, $B_{z}(r, z=$ $\pm L / 2)=0$, we obtain that $k_{n}=(2 n-1) \pi / L$. The magnetic potentials can then be written as a sum over $n$ :

$$
\begin{aligned}
& \phi_{\text {inner }}(r, z)=\sum_{n=1}^{\infty} \alpha_{n} I_{0}\left(k_{n} r\right) \sin \left(k_{n} z\right), \\
& \phi_{\text {outer }}(r, z)=\sum_{n=1}^{\infty} \sin \left(k_{n} z\right)\left[\beta_{n} I_{0}\left(k_{n} r\right)+\gamma_{n} K_{0}\left(k_{n} r\right)\right] .
\end{aligned}
$$

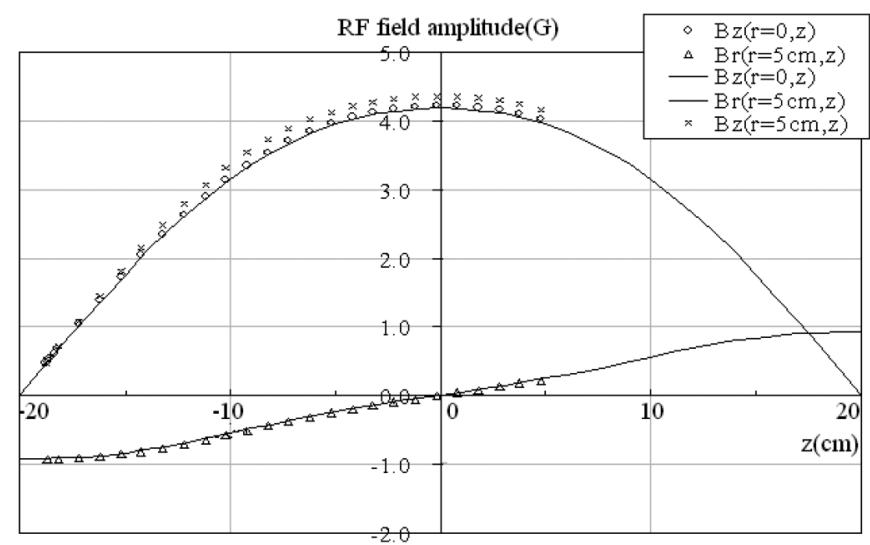

FIG. 5. Measured $(\bigcirc, \times$, and $\triangle)$ and calculated field components (solid lines) for $B_{z}(r=0 \mathrm{~cm}, z), B_{z}(r=5 \mathrm{~cm}, z)$, and $B_{r}(r=5 \mathrm{~cm}, z)$ of the RFSR. The solenoid is $30 \mathrm{~cm}$ long and the RFSR, i.e., the rf field, is $40 \mathrm{~cm}$ long.

Next, we impose two additional boundary conditions: $B_{r}(r=S, z)$ must be continuous and $B_{r}(r=R, z)=0$, where $S$ is the radius of the solenoid and $R$ is the radius of the cylindrical aluminum shield. These two conditions determine $\beta_{n}$ and $\gamma_{n}$. The boundary condition $\frac{\mathrm{d}}{\mathrm{d} z} \times$ $\left[\phi_{\text {outer }}(S, z)-\phi_{\text {inner }}(S, z)\right]=J$, where $J$ is the current density of the solenoid, determines $\alpha_{n}$. By using the orthogonality of the $g_{n}(z)$, we obtain

$$
\begin{aligned}
J \int_{-M / 2}^{+M / 2} \cos \left(k_{n} z\right) \mathrm{d} z= & k_{n} \int_{-L / 2}^{+L / 2} \cos ^{2}\left(k_{n} z\right) \mathrm{d} z \\
& \times\left[\left(\beta_{n}-\alpha_{n}\right) I_{0}\left(k_{n} S\right)+\gamma_{n} K_{0}\left(k_{n} S\right)\right],
\end{aligned}
$$

and after integration

$$
\frac{2 J \sin \left(k_{n} M / 2\right)}{k_{n}}=\frac{k_{n} L}{2}\left[\left(\beta_{n}-\alpha_{n}\right) I_{0}\left(k_{n} S\right)+\gamma_{n} K_{0}\left(k_{n} S\right)\right],
$$

where $M$ is the length of the solenoid and $L$ the length of the aluminum shield. Figure 5 shows the calculated fields which are a sum of the first 40 terms of the slowly converging series.

\section{Measurement of the rf fields}

The field of the RFSR was measured to verify the results of the calculations. A small probe coil was fabricated and mounted on an arm so that the axis of the coil was either parallel or perpendicular to the axis of the RFSR. The probe was then stepped by a computerized scanner to map the rf field of the RFSR. The probe coil has 16 turns of enamel-coated $\mathrm{Cu}$ wire on a diameter of $7.8 \mathrm{~mm}$. All the other components of the probe system were made of plastic. It was important to make sure that the axis of the probe coil was accurately perpendicular to the axis of the rf field since the radial field component $B_{r}$ is much smaller than the axial field component $B_{z}$ which could easily contaminate the measurement of the radial field component. For 
the same reason, the coil of the probe was carefully wound so that each turn was perpendicular to the axis of the coil.

A $100 \mathrm{mV}_{\mathrm{PP}}$ sine wave, corresponding to $1 \mathrm{~A}$ current in the solenoid, at $29 \mathrm{kHz}$ was connected to the reference input of a lock-in amplifier and to the input of the audio amplifier which was driving the series tuned $L C$ circuit of the RFSR. The signal from the probe coil was then connected to the input of the lock-in amplifier. The amplitude of the rf field was obtained from the root-mean-squared output voltage of the lock-in amplifier.

The $B_{z}$ and $B_{r}$ components at $r=0 \mathrm{~cm}$ and $5 \mathrm{~cm}$ were mapped with $10-\mathrm{mm}$ steps. The maximum of $B_{z}$ on axis was measured to be $0.42 \mathrm{mT}$ at the center of the solenoid and increasing slowly with $r$. On the axis $B_{r}=0 \mathrm{mT}$ and off axis $B_{r}$ increases when approaching the ends of the solenoid. Neither field components were distorted much by the aluminum windows. Figure 5 shows the measured $B_{z}$ at $r=0 \mathrm{~cm} \mathrm{(O)} \mathrm{and} r=5 \mathrm{~cm} \mathrm{( \times ),} \mathrm{and} B_{r}$ at $r=5 \mathrm{~cm} \mathrm{( \triangle )}$ with the corresponding calculated fields (solid lines), agreement between the measured and calculated fields is within $1 \%$.

\section{SPIN-FLIP EFFICIENCY OF THE RF SPIN ROTATOR}

We have developed two independent methods to evaluate the neutron spin-flip efficiency of the RFSR over the phase space defined by the $1 F P 12$ neutron guide. In the NPDGamma experiment the spin-flip efficiency is one of the parameters required to extract the final parity violating physics $\gamma$-ray asymmetry

$$
A_{\gamma} \equiv \frac{A_{\gamma}^{\text {measured }}}{P_{n} \cdot \varepsilon \cdot c} .
$$

Here $P_{n}$ is neutron beam polarization, $\varepsilon$ is the RFSR efficiency, and the constant $c$ contains the rest of the corrections for $A_{\gamma}^{\text {measured }}$ as discussed in Refs. [19,21].

The spin-flip efficiency measurements consisted of a set of neutron transmission measurements with monitor M1, polarizer (P), M2, RFSR, analyzer (A), and M3 (see Fig. 1). The neutron beam was first polarized by the ${ }^{3} \mathrm{He}$ spin filter with opacity $x_{\mathrm{P}}=n_{\mathrm{P}} \sigma l_{\mathrm{P}}$ and ${ }^{3} \mathrm{He}$ polarization of $P_{\mathrm{P}}$. Here $n_{\mathrm{P}}$ is the ${ }^{3} \mathrm{He}$ number density, $l_{\mathrm{P}}$ the length of the ${ }^{3} \mathrm{He}$, and $\sigma$ is the spin-dependent $n-{ }^{3} \mathrm{He}$ absorption cross section for the reaction channel $J=0$. The cross section $\sigma$ has the well-known $1 / v$-energy dependence, where $v$ is the neutron speed. After the spin filter the polarized neutrons enter the RFSR where the spin direction of beam was rotated by $\pi$ radians with efficiency $\varepsilon, 0 \leq \varepsilon \leq 1$. If the RFSR was off, there was no change to the spin direction. After passing the RFSR, the polarization of the beam was analyzed by the polarization analyzer. After the polarization analyzer the transmitted neutrons were detected by monitor M3. The analyzer cell had opacity $x_{\mathrm{A}}=n_{\mathrm{A}} \sigma l_{\mathrm{A}}$, where $n_{\mathrm{A}}$ is the ${ }^{3} \mathrm{He}$ number density, $l_{\mathrm{A}}$ is the thickness of the ${ }^{3} \mathrm{He}$, and the ${ }^{3} \mathrm{He}$ polarization in the cell is $P_{\mathrm{A}}$.
We used two different neutron transmission methods to measure the spin-flip efficiency. In the RFSR-flip method the efficiency measurement was obtained by comparing the RFSR on and off transmissions. In the AFP ${ }^{3} \mathrm{He}$ flip method, the transmission through the RFSR was measured before and after the neutron beam polarization was flipped by an AFP flip of the ${ }^{3} \mathrm{He}$ polarization in the spin filter cell.

\section{A. Spin-flip efficiency using the RFSR-flip method}

In the RFSR-flip method, the polarizer and analyzer have fixed polarization directions and the spin-flip efficiency is obtained from the ratio of the transmission through the analyzer with the RFSR on and off. Let $x_{\mathrm{A}}$ and $x_{\mathrm{P}}$ be the opacities of the analyzer and polarizer and $P_{\mathrm{A}}$ and $P_{\mathrm{P}}$ the ${ }^{3} \mathrm{He}$ polarizations of the analyzer and polarizer. The number of neutrons in the two spin states ( \pm states corresponds to up/down states) incident on the analyzer when the RFSR is off is

$$
N_{\mathrm{P}}^{ \pm}=\frac{N_{0}}{2} T_{\mathrm{P}}^{ \pm}=\frac{N_{0}}{2} e^{-x_{\mathrm{P}}\left(1 \mp P_{\mathrm{P}}\right)},
$$

assuming no loss of neutrons after the polarizer. Here $N_{0}$ is the total number of unpolarized neutrons entering the polarizer. The total number of neutrons in the two spin states, arriving at the analyzer cell when the RFSR is off, is then

$$
N_{\mathrm{P}}=N_{\mathrm{P}}^{+}+N_{\mathrm{P}}^{-}=N_{0} e^{-x_{\mathrm{P}}} \cosh \left(x_{\mathrm{P}} P_{\mathrm{P}}\right) .
$$

The number of the transmitted neutrons through the analyzer cell is

$$
N_{\mathrm{off}-\mathrm{A}}^{ \pm}=N_{\mathrm{P}}^{ \pm} e^{-x_{\mathrm{A}}\left(1 \mp P_{\mathrm{A}}\right)}
$$

and thus the total number of neutrons after the analyzer, when the RFSR is off, is

$$
\begin{aligned}
N_{\text {off-A }} & =N_{\text {off-A }}^{+}+N_{\text {off-A }}^{-} \\
& =N_{0} e^{-\left(x_{\mathrm{A}}+x_{\mathrm{P}}\right)} \cosh \left(x_{\mathrm{A}} P_{\mathrm{A}}+x_{\mathrm{P}} P_{\mathrm{P}}\right) .
\end{aligned}
$$

When the RFSR is on, the beam polarization $\left(P_{n}\right)$ rotates $\pi$ radians about the $\hat{z}$ axis while the neutrons traverse through the RFSR. The spin reversal is not perfect and we define the spin rotation efficiency $\varepsilon$,

$$
P_{n-\mathrm{on}} \equiv(1-2 \varepsilon) P_{n \text {-off }},
$$

where $0 \leq \varepsilon \leq 1$ and $P_{n \text {-off }}=\tanh \left(x_{\mathrm{P}} P_{\mathrm{P}}\right)$. After the RFSR the number of neutron in the two spin states are

$$
\begin{aligned}
& N_{\mathrm{P}-\text { on }}^{+}=(1-\varepsilon) \frac{N_{0}}{2} e^{-x_{\mathrm{P}}\left(1-P_{\mathrm{P}}\right)}+\varepsilon \frac{N_{0}}{2} e^{-x_{\mathrm{P}}\left(1+P_{\mathrm{P}}\right)} \\
& N_{\mathrm{P}-\text { on }}^{-}=(1-\varepsilon) \frac{N_{0}}{2} e^{-x_{\mathrm{P}}\left(1+P_{\mathrm{P}}\right)}+\varepsilon \frac{N_{0}}{2} e^{-x_{\mathrm{P}}\left(1-P_{\mathrm{P}}\right)} .
\end{aligned}
$$

The transmission of the neutrons of the known spin states through the analyzer cell is 


$$
N_{\mathrm{on}-\mathrm{A}}^{+}=N_{\mathrm{P}-\mathrm{on}}^{+} e^{-x_{\mathrm{A}}\left(1-P_{\mathrm{A}}\right)} \quad N_{\mathrm{on}-\mathrm{A}}^{-}=N_{\mathrm{P}-\mathrm{on}}^{-} e^{-x_{\mathrm{A}}\left(1+P_{\mathrm{A}}\right)} .
$$

The total number of neutrons which pass through the analyzer is

$$
\begin{aligned}
N_{\text {on- } \mathrm{A}}= & N_{0} e^{-\left(x_{\mathrm{A}}+x_{\mathrm{P}}\right)}\left[(1-\varepsilon) \cosh \left(x_{\mathrm{A}} P_{\mathrm{A}}+x_{\mathrm{P}} P_{\mathrm{P}}\right)\right. \\
& \left.+\varepsilon \cosh \left(x_{\mathrm{A}} P_{\mathrm{A}}-x_{\mathrm{P}} P_{\mathrm{P}}\right)\right] .
\end{aligned}
$$

The transmitted neutrons through the analyzer, when the RFSR is off and on, are given by Eqs. (5.4) and (5.8). Let $\mathrm{M} 3_{\text {off(on) }}$ be the monitor signals from neutron transmission through the analyzer with the RFSR off and on. The efficiency of the RFSR, $\varepsilon$, can be now extracted from the measured transmission ratio

$$
\frac{\mathrm{M} 3_{\text {on }}}{\mathrm{M} 3_{\text {off }}}=\frac{(1-\varepsilon) \cosh \left(x_{\mathrm{A}} P_{\mathrm{A}}+x_{\mathrm{P}} P_{\mathrm{P}}\right)+\varepsilon \cosh \left(x_{\mathrm{A}} P_{\mathrm{A}}-x_{\mathrm{P}} P_{\mathrm{P}}\right)}{\cosh \left(x_{\mathrm{A}} P_{\mathrm{A}}+x_{\mathrm{P}} P_{\mathrm{P}}\right)},
$$

assuming that parameters $x_{\mathrm{A}}, x_{\mathrm{P}}, P_{\mathrm{A}}$, and $P_{\mathrm{P}}$ are known.

\section{B. Spin-flip efficiency using the AFP ${ }^{3} \mathrm{He}$ flip method}

A nearly perfect reversal of neutron beam polarization can be achieved by performing a ${ }^{3} \mathrm{He}$ polarization reversal in the polarizer by AFP. After an AFP flip, the analyzed beam polarization can be compared to the RFSR flipped beam polarization to determine the beam polarization reversal efficiency $\varepsilon$. To derive the number of neutrons entering the monitor M3 after the AFP, we need only to change the sign of the ${ }^{3} \mathrm{He}$ polarization $P_{\mathrm{P}}$ in the polarizer, in Eq. (5.2),

$$
N_{\overline{\mathrm{AFP}}}^{ \pm}=\frac{N_{0}}{2} T_{\mathrm{P}}^{ \pm}=\frac{N_{0}}{2} e^{-x_{\mathrm{P}}\left(1 \pm P_{\mathrm{P}}\right)},
$$

and then transmit these neutrons through the analyzer and compare the result with the result from the RFSR flip given by Eq. (5.8). The total number of the AFP flipped neutrons incident on M3 is

$$
N_{\mathrm{AFP}}=N_{\mathrm{AFP}}^{+}+N_{\mathrm{AFP}}^{-}=N_{0} e^{-\left(x_{\mathrm{A}}+x_{\mathrm{P}}\right)} \cosh \left(x_{\mathrm{A}} P_{\mathrm{A}}-x_{\mathrm{P}} P_{\mathrm{P}}\right) .
$$

The RFSR efficiency can be then extracted from the measured transmission signals $\mathrm{M} 3_{\text {off }}, \mathrm{M} 3_{\text {on }}$, and $\mathrm{M} 3_{\mathrm{AFP}}$ :

$$
\frac{N_{\text {off-A }}-N_{\text {on-A }}}{N_{\text {off-A }}-N_{\text {AFP }}}=\frac{\mathrm{M} 3_{\text {off }}-\mathrm{M} 3_{\text {on }}}{\mathrm{M} 3_{\text {off }}-\mathrm{M} 3_{\mathrm{AFP}}}=\varepsilon
$$

\section{Measurement, analysis, and results of the spin-flip efficiency}

In Eqs. (5.9) and (5.12), the determination of the spinflip efficiency using the polarizer and the analyzer cells depends on ${ }^{3} \mathrm{He}$ polarization and ${ }^{3} \mathrm{He}$ thickness in the cells seen by neutron beam through the parameters $x_{\mathrm{A}}, x_{\mathrm{P}}, P_{\mathrm{A}}$, and $P_{\mathrm{P}}$. All of these parameters were constant during the measurements except $P_{\mathrm{A}}$, which varied slowly with time. The ${ }^{3} \mathrm{He}$ spin filter was continuously optically pumped to stabilize the polarization at $46 \pm 2 \%$ as determined from relative neutron transmission measurements. The description of the spin filter and its performance are given in
Ref. [17]. The analyzer cell was a $2.5 \mathrm{~cm}$ diameter sphere which was polarized outside of the experiment using a single bar diode laser with its spectrum narrowed by an external cavity [22]. For each spin-flip efficiency measurement, the polarization of the analyzer was measured with NMR, the maximum analyzer polarization was observed to be $61 \pm 5 \%$. The polarized analyzer cell was then transported to the experiment in a few gauss magnetic field to minimize depolarization: $1 \%-2 \%$ polarization was typically lost during the operation. The decay time constant of the polarization in the room temperature cell was measured to be 130 hours. After the neutron spin-flip efficiency measurements, the cell was returned to the spin-exchange optical pumping system and the polarization was remeasured by NMR. The NMR results were also verified by neutron transmission measurements. The largest source of systematic error in determining the ${ }^{3} \mathrm{He}$ polarization of the analyzer cell by neutron transmission was uncertainty in the effective ${ }^{3} \mathrm{He}$ thickness from the curved GE180 glass walls of this spherical cell. The systematic uncertainties involved using the ${ }^{3} \mathrm{He}$ polarizer are discussed in Ref. [17].

In the analysis of the spin-flip efficiency data, each data set consisted of signal runs and their corresponding background runs taken with the beam shutter closed. A single run contained 1000 neutron pulses. The monitor M3 data was normalized with the M1 data and then background runs were subtracted. The efficiencies were calculated by integrating the monitor signals over the neutron energy range of 3-18 meV. The lower energy side of this range was defined by an upstream neutron chopper that started closing off the beam at $30 \mathrm{~ms}$ for background measurements from 34 to $50 \mathrm{~ms}$. Figure 6 shows the measured neutron spectra of M1, M2, and M3 as a function of neutron time of flight, with M2 and M3 signals multiplied by a factor of three and two, respectively. Because of the $1 / v$ dependence of the ${ }^{3} \mathrm{He}$-neutron absorption cross section, more beam attenuation takes place at low neutron energies. The ${ }^{3} \mathrm{He}$ thickness in M1 and M2 is about equal but in M3 has significantly more ${ }^{3} \mathrm{He}$. Figure 7 shows M3 signals as a function of time of flight for eight consecutive spin states $\downarrow \uparrow \uparrow \downarrow \uparrow \downarrow \uparrow$ where $\downarrow(\uparrow)$ represents the RFSR off-state (on-state). 


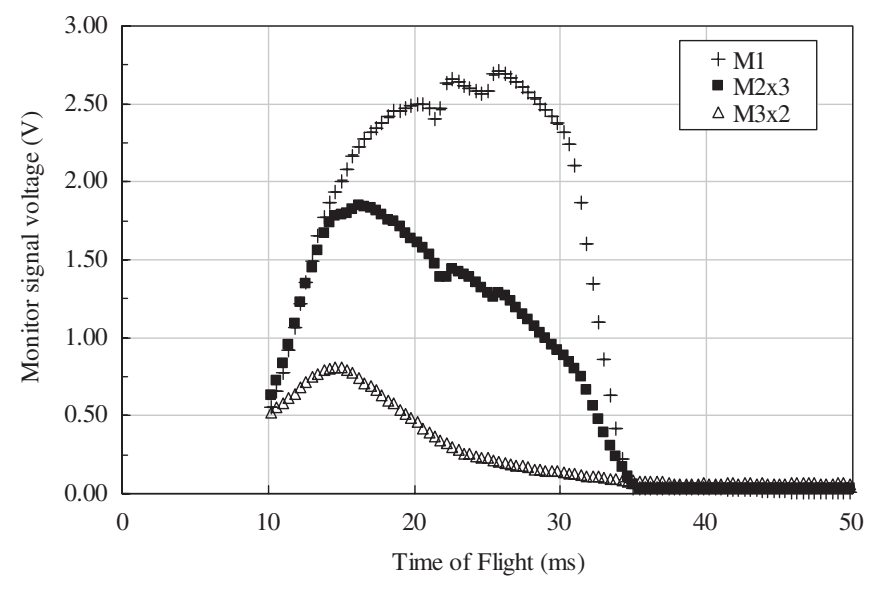

FIG. 6. Signals from the monitors M1, M2, and M3 as a function of neutron time-of-flight. M1 and M2 have the same ${ }^{3} \mathrm{He}$ thickness and preamplifier gains, whereas M3 has significantly more ${ }^{3} \mathrm{He}$ and about 50 times larger gain. The difference between M1 and M2 signals is caused mainly by the ${ }^{3} \mathrm{He}$ spin filter located between M1 and M2 and the $1 / v$ dependence of the $n-{ }^{3} \mathrm{He}$ absorption cross section that causes more beam attenuation at low neutron energies. The signals have been shifted in time to start at the same time to make the comparison easier.

Figure 8 shows two superimposed measured efficiencies as a function of the relative $\mathrm{rf}$ field change, $\Delta B_{1} / B_{1}$. Symbols $(\triangle)$ represent data measured using the RFSRflip method of Eq. (5.9) and the data points $(\bullet)$ are measured with the AFP ${ }^{3} \mathrm{He}$ flip method of Eq. (5.12). In the measurement the static field is set to correspond to the maximum RFSR efficiency value, i.e., to the resonance condition of Eq. (2.1). The lines are fits to data using quadratic functions. Results from both measurements are in good agreement and the maximum efficiencies with each method are the same within the experimental error.

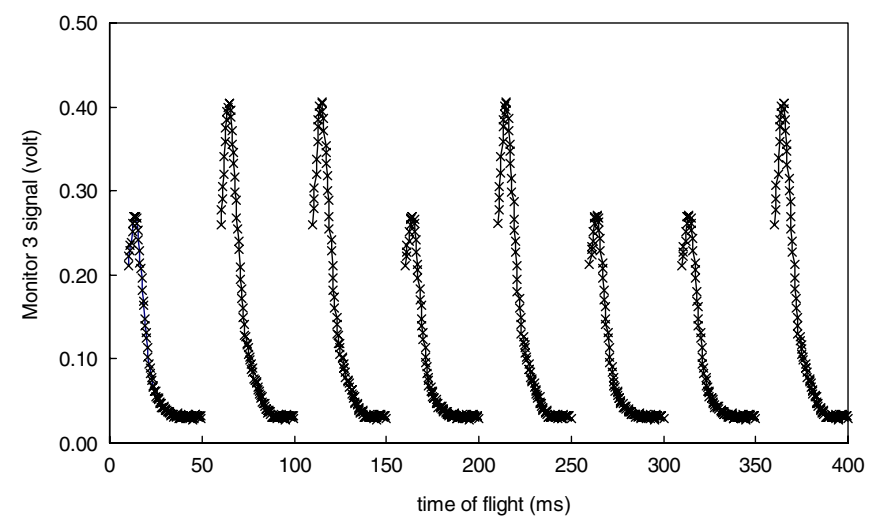

FIG. 7. Eight consecutive neutron pulses corresponding to the eight-step spin sequence ( $\downarrow \uparrow \uparrow \downarrow \uparrow \downarrow \uparrow)$ of the RFSR measured by M3 as a function of time of flight. Each neutron time-of-flight frame is $50 \mathrm{~ms}$ long. Beam absorption in the ${ }^{3} \mathrm{He}$ analyzer cell is much larger when the beam polarization is opposite to the ${ }^{3} \mathrm{He}$ polarization. Here $\downarrow(\uparrow)$ represents the RFSR off-state (on-state).

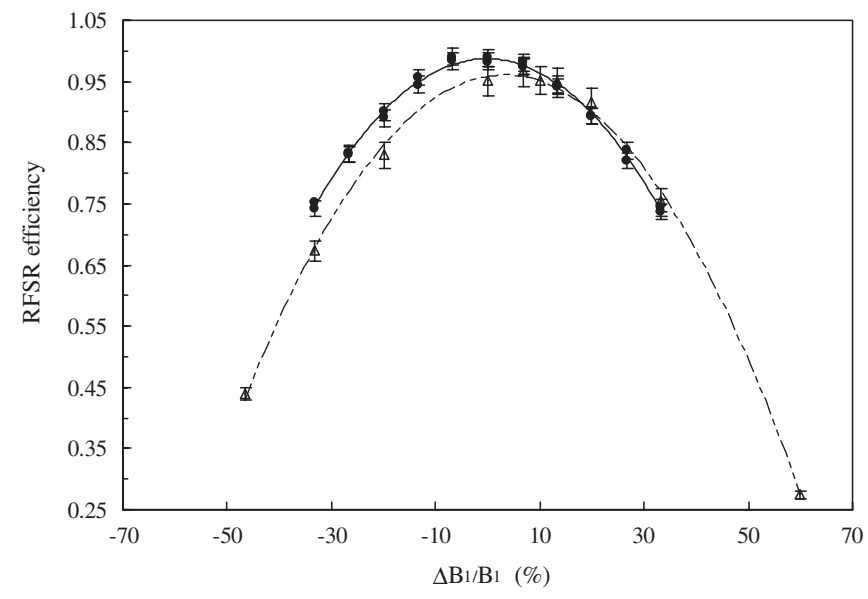

FIG. 8. The measured efficiencies of the RFSR using the RFSR-flip method $(\triangle)$ and the AFP ${ }^{3} \mathrm{He}$ flip method $(\bullet)$ are plotted as a function of a relative change in the rf field, $\Delta B_{1} / B_{1}$. The dashed and solid curves are quadratic fits to data.

Figure 9 presents measured efficiencies as a function of a relative change in the static field, $\Delta B_{0} / B_{0}$ when $B_{1}$ is kept fixed at the maximum RFSR efficiency. The data set $(\Delta)$ is measured using the RFSR-flip method and the data set (•) using the AFP ${ }^{3} \mathrm{He}$ flip method. Each data set is fitted to a quadratic function.

In order to determine the spin-flip efficiency over the entire phase space of the beam, the rf field amplitude scan was performed on three off-axis analyzer positions: one in the vertical position $(x=0, y=3.3 \mathrm{~cm})$ and two in the horizontal positions ( $x= \pm 3.3 \mathrm{~cm}, y=0)$, and then efficiencies were measured using the RFSR-flip method. Results are presented in Fig. 10. The three data sets are fitted to quadratic functions: the short-dashed line corresponds to $x=+3.3 \mathrm{~cm}$, the medium-dashed line to

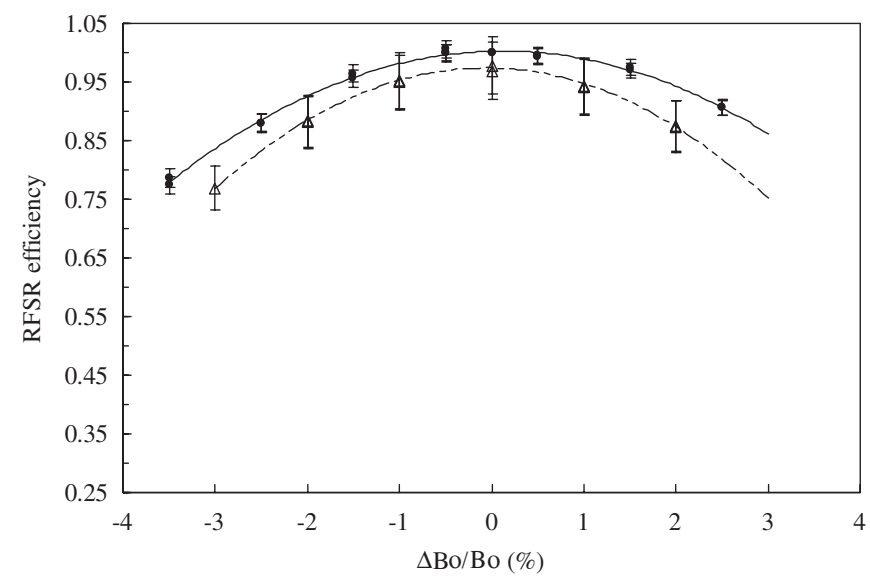

FIG. 9. Measured efficiencies of the RFSR are shown in a function of a relative change in the static magnetic field. Triangles are measured with the RFSR-flip method and solid points with the AFP ${ }^{3} \mathrm{He}$ flip method. Lines are results of quadratic fits to the data set. 


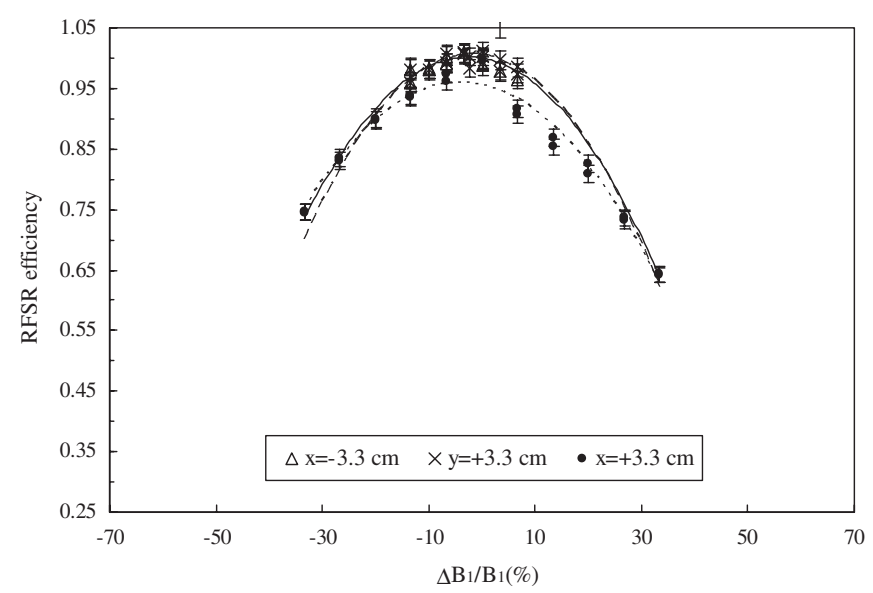

FIG. 10. The spin-flip efficiency of the RFSR on off-axis at $x= \pm 3.3 \mathrm{~cm}$ (circle and triangle) and $y=+3.3 \mathrm{~cm}$ (cross) as a function of the rf field amplitude $B_{1}$. The data are measured using the RFSR-flip method. The lines (short-dashed, mediumdashed, long-dashed lines) are quadratic fits to the data set.

$x=-3 \mathrm{~cm}$, and the long-dashed line to the $y=+3 \mathrm{~cm}$ position.

Near maximum the values of the $x=+3.3 \mathrm{~cm}$ position seem systematically lower than the values of the other positions. The maximum efficiency values given by the fits for position of $x=y=0$ in Fig. 8 and for positions of $x= \pm 3.3, y=0$ and $x=0, y=+3.3$ in Fig. 10 were averaged for determination of the overall efficiency across the beam. The overall spin-flip efficiency across the beam was $98.8 \pm 0.5 \%$.

In the RFSR-flip method the dominating systematic uncertainty is the accuracy of the ${ }^{3} \mathrm{He}$ thickness and ${ }^{3} \mathrm{He}$ polarization; $x_{\mathrm{A}}$ and $P_{\mathrm{A}}$ for the analyzer and $x_{\mathrm{P}}$ and $P_{\mathrm{P}}$ for the polarizer. These parameters were determined by performing auxiliary neutron transmission measurements. The relative uncertainties in $x_{\mathrm{A}}$ and $P_{\mathrm{A}}$ are 0.02 and 0.04 , and in $x_{\mathrm{P}}$ and $P_{\mathrm{P}}$ are 0.02 and 0.03 , respectively. The length of the flight path needed in the determination of the neutron energy was measured by using the accurately known Bragg edges on beryllium, where the total neutron cross section changes discontinuously as a function of neutron energy. The relative error in the flight path length is $2 \times 10^{-3}$ [22]. To find the maximum efficiency, data in Figs. 8-10 were fitted with a quadratic form for a spin-flip probability described in Eq. (2.1) which introduced additional error of 0.01 . The overall systematic error in the efficiency when using the RFSR spin-flip method is less than 5\%. In the AFP ${ }^{3} \mathrm{He}$ flip method where the knowledge of the polarization and the thickness of the ${ }^{3} \mathrm{He}$ are not required, the systematic error is $<2 \%$. The figures show only the statistical uncertainties.

Because of thermalization processes in the moderator, geometric effects such as the time distribution of neutrons from the neutron moderator, path length differences, neutron beam divergence, and small changes in the kinetic energy of the neutrons by the RFSR for off-resonance neutrons, there is a distribution of spin rotation angles around $\pi$ radians in the RFSR. The fractional width of this distribution is estimated to be at the $1 \%$ level. This departure of the spin-flip probability from unity translates into a $10^{-4}$ effect in the spin-flip efficiency.

\section{Measurement of the spin-flip efficiency with parity- violating $\gamma$-ray asymmetry on ${ }^{35} \mathrm{Cl}$}

As an independent determination of the performance of the RFSR, we measured the parity-violating $\gamma$-ray asymmetry $A_{\gamma}$ from polarized neutron capture on ${ }^{35} \mathrm{Cl}$ as a function of the relative change in the rf field amplitude $B_{1}$. The $\gamma$ rays were detected using the NPDGamma current-mode $\mathrm{CsI}(\mathrm{Tl}) \quad \gamma$-detector array, described in Ref. [19]. At low neutron energies, $A_{\gamma}$ on ${ }^{35} \mathrm{Cl}$ is measured to be large $(26 \pm 7) \times 10^{-6}[23]$.

Using parity-violating $A_{\gamma}$ as a beam polarization analyzer, the efficiency $\varepsilon$ can be calculated from Eq. (5.1) if beam polarization $P_{n}$ and the instrumental constant $c$ are accurately known. Figure 11 shows a result of such a measurement. A small geometrical difference between the two measurements of Figs. 8 and 11 is that the $\mathrm{Cl}$ measurement was performed with $5 \mathrm{~cm}$ beam collimation compared to the $1.3 \mathrm{~cm}$ collimation of the ${ }^{3} \mathrm{He}$ analyzer. The systematic errors present in $\mathrm{Cl}$ measurement and in the neutron transmission measurement are completely different.

To reach $20 \%$ precision in the spin-flip efficiency measurement with the Cl-target required a few hours on 1FP12 at LANSCE. At the higher flux neutron beams which will soon be available at SNS and JSNS, the use of the parityviolating $\gamma$-ray asymmetry $A_{\gamma}$ on ${ }^{35} \mathrm{Cl}$ can be a fast and

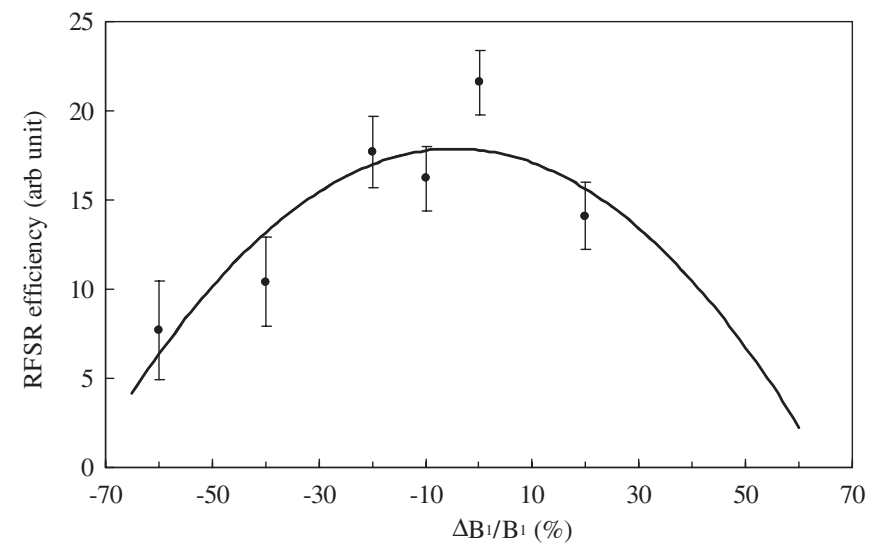

FIG. 11. The spin-flip efficiency of the RFSR is shown as a function of a relative change in the rf field $\Delta B_{1} / B_{1}$. This spinflip efficiency measurement with cold neutrons is based on the well-known parity-violating $\gamma$-ray asymmetry on ${ }^{35} \mathrm{Cl}$ at cold neutron energies (see text). The solid line is a fit to a quadratic function. 
effective method of neutron polarization analysis of transmitted beams.

\section{SYSTEMATIC EFFECTS PRODUCED BY THE RFSR ON THE NPDGAMMA EXPERIMENT}

Since the NPDGamma experiment aims to measure the $\gamma$ asymmetry with a precision of $1 \times 10^{-8}$, false asymmetries have to be controlled to an even greater accuracy. Here we consider systematic effects caused by the RFSR on the measured $A_{\gamma}$ in the $n-p$ capture reaction at cold neutron energies. For detection of a false asymmetry, a signal has to be produced which is in phase with the state of the neutron spin. Otherwise it will average out with time and appear as another source of random noise. The origin of these systematic effects can be either instrumental or due to neutron interactions with matter other than $n-p$ capture in the liquid parahydrogen target. The physics origins of the systematic effects have been discussed in detail in Ref. [9] and will not be repeated here. Since the parity-violating asymmetry $A_{\gamma}$ is energy independent over the range of neutron energies in this experiment, systematic effects, which in many cases have energy dependence [9], can be isolated using accurate neutron time of flight.

The most serious potential source of an instrumental systematic effect is the rf field of the spin rotator. It is perfectly coherent with the neutron spin state and there is an electronic difference in the RFSR between the spin-up and spin-down states. To minimize any potential problem from electronic pickup, the rf power level is held constant and switched either to the RFSR or to a dummy load (see Sec. IV C).

\section{A. Raw parity-violating $\boldsymbol{\gamma}$-ray asymmetry}

The $48 \mathrm{CsI}(\mathrm{Tl})$ crystals of the $\gamma$-ray detector array are symmetrically mounted around the target consisting of four rings of 12 detectors per ring. The detector array is divided into symmetric upper and lower detector pairs [19]. This allows the experiment to measure $A_{\gamma}$ in single neutron pulse (in single spin state)

$$
A_{\gamma}^{\text {measured }}=\frac{U_{\uparrow}-D_{\uparrow}}{U_{\uparrow}+D_{\uparrow}},
$$

where $U_{\uparrow}\left(D_{\uparrow}\right)$ is the sum of the signal of the upper (lower) detector pairs when the neutron spin is up. The $\gamma$-ray asymmetry $A_{\gamma}$ can be determined from $A_{\gamma}^{\text {measured }}$ after applying corrections for beam polarization, RFSR spinflip efficiency, neutron depolarization in target, and detector acceptance [see Eq. (5.1)]. Since the gains of the upper and lower detector pairs are difficult to equalize to $\mathrm{ppm}$ accuracy, we switch between the upper and lower detector pairs by reversing the neutron spin direction by the RFSR. The raw asymmetry is thus a difference between the two spin states $[19,21]$ :

$$
A_{\gamma}^{\text {measured }}=\frac{U_{\uparrow}-D_{\uparrow}-\left(U_{\downarrow}-D_{\downarrow}\right)}{U_{\uparrow}+D_{\uparrow}+U_{\downarrow}+D_{\downarrow}}
$$

\section{B. Additive and multiplicative coupling of $\mathrm{rf}$ power of the RFSR to the detector system}

A design requirement of the NPDGamma experiment is that it must be possible to measure any electronic effects resulting in a false asymmetry to the same accuracy desired for $A_{\gamma}$ in a time short compared to the measurement time of the experiment. Instrumental systematic effects caused by the RFSR have been extensively tested for and are described in Ref. [19].

An additive effect, in which a false asymmetry may be induced as a result of an additional signal in the detector electronics, can be produced by pickup of the spin rotator signal. The additive effects have been searched for by measuring $A_{\gamma}$ without the incident neutron beam but operating the RFSR. Because of a small width of the distribution of the electrical noise, we can measure $A_{\gamma}$ in a few hours to an accuracy of $10^{-9}$ [19]. The noise observed in the detectors, with the spin rotator running, did not significantly change from the measured and calculated detector noise in the 48 detectors, which includes contributions from dark currents and cosmic-ray background [19,20].

A multiplicative effect is a gain change in the detector system, caused, for example, by a spin rotator magnetic field leakage into the detector system and a consequent gain change. A search for multiplicative effects is accomplished by using the two LEDs per crystal to simulate light intensities expected to be produced by neutrons during an actual measurement [19].

For the $10^{-9}$ accuracy in $A_{\gamma}$, we needed 3-4 times more time to perform a search of multiplicative effects compared to the additive effects. The longer run time required is caused by the calibration light introduced by LEDs which has greater noise than the electrical noise.

These measurements have shown that the shielding of the RFSR is sufficient to electronically isolate the RFSR power from the rest of the experiment in the sensitivity level of $10^{-9}$ [19].

\section{Control of drifts by an eight-step spin sequence}

To control systematic effects caused by time-dependent drifts in detector efficiencies, we use a symmetric eightstep spin sequence, $\downarrow \uparrow \uparrow \downarrow \uparrow \downarrow \downarrow \uparrow$ where $\downarrow$ ( $\uparrow$ represents the RFSR off-state (on-state), or its complement. The four spin-up states are added together to obtain $U_{\uparrow}$ and $D_{\uparrow}$ and then the other four to obtain $U_{\downarrow}$ and $D_{\downarrow}$. The asymmetry $A_{\gamma}^{\text {measured }}$ is calculated for each eight-state sequence. Use of signals $(S)$ of such spin-state octets cancels both linear drifts (e.g. $+S-2 S-3 S+4 S-5 S+6 S+7 S-8 S=0) \quad$ and quadratic drifts (e.g. $+S-4 S-9 S+16 S-25 S+$ $36 S+49 S-64 S=0)$ over the octet. 


\section{Other systematic effects on the NPDGamma produced by the RFSR}

Two additional sources of systematic effects are considered. One is a change in the kinetic energy of a neutron caused by the RFSR. As discussed in Sec. II this is possible only when the static field is different in the entry of the RFSR compared to the exit. We know from the field measurement that the field difference is less than $0.2 \mu \mathrm{T}$ across the RFSR, then the relative change in the kinetic energy of a 4-meV neutron is less than $10^{-5}$ leading to the second order effects on $A_{\gamma}$ which are less than $10^{-10}$.

To estimate the effect of the beam up and down motion by the Stern-Gerlach steering, we used the measured result for the field gradient $\frac{\partial B_{y}}{\partial z} \leq 10 \mu \mathrm{T} / \mathrm{m}$ between the RFSR and the end of the $\mathrm{LH}_{2}$ target. The small difference between the beam positions in the detector with the neutron spin-up state and spin-down state leads to a $10^{-10}$ change in the solid angle of the detector.

\section{CONCLUSION}

The NPDGamma experiment aims to determine the pion contribution $f_{\pi}^{1}$ in the parity-violating interaction between nucleons by measuring the parity-violating $\gamma$-ray asymmetry $A_{\gamma}$ with the accuracy of $1 \times 10^{-8}$ in the polarized cold neutron capture reaction in parahydrogen. The experiment has to control systematic uncertainties below the $10^{-8}$ level. For this resonant rf spin rotator to reverse the neutron polarization in a pulsed cold beam was developed. The efficiency of the spin rotator was measured to be $98.8 \pm$ $0.5 \%$ for neutron energies from 3.3 to $18.4 \mathrm{meV}$ over the full phase space of the beam, which possesses a $9.5 \mathrm{~cm} \times$ $9.5 \mathrm{~cm}$ cross sectional area and a transverse momentum spread set by a $19 \mathrm{~m}$ long $m=3$ supermirror neutron guide as described and characterized in Ref. [14]. We have placed upper bounds which are less than a few times $10^{-9}$ on the size of the systematic effects that the rf spin rotator introduces into the NPDGamma experiment. The spin rotator has already been successfully used in a number of measurements of parity violation in polarized neutron capture in several nuclei with mass $A \geq 27$ [21] and in the NPDGamma experiment that had the first phase data run at LANSCE in 2006.

We note that many of the properties of both the resonant rf spin rotator itself and the techniques used to characterize its efficiency in this work are also applicable to future sensitive polarized neutron experiments using pulsed cold neutron beams. Since the rotator operates in a uniform static magnetic field, it is fully compatible with the small magnetic field gradient requirements of polarized ${ }^{3} \mathrm{He}$ neutron spin filters. As the performance of ${ }^{3} \mathrm{He}$ neutron spin filters continues to improve [17] their use in neutron scattering spectrometers promises to expand, and it is possible that their broad phase space acceptance and wide dynamic range in neutron energy can make possible new types of neutron scattering spectrometers. For many such instruments in the future, it is likely that a resonant $\mathrm{rf}$ spin rotator will be an attractive choice for neutron spin reversal. The combined use of polarized ${ }^{3} \mathrm{He}$ neutron polarizers and analyzers and $\mathrm{rf}$ spin rotators is proposed in the polarized neutron $\beta$-decay experiments at SNS [24], where the unique properties of ${ }^{3} \mathrm{He}$ neutron spin filters are well suited to absolute determination of the neutron beam polarization.

\section{ACKNOWLEDGMENTS}

The authors would like to thank Mr. G. Peralta for his technical contribution to the success of this work. The work was supported in part by the U.S. Department of Energy (Office of Energy Research, under Contract No. W7405-ENG-36), the National Science Foundation (Grants No. PHY-0100348 and No. PHY-0457219), the Natural Science and Engineering Research Council of Canada, and the Japanese Grant-in-Aid for Scientific Research A12304014. M. Snow thanks the Institute for Nuclear Theory at the University of Washington for its hospitality and the Department of Energy for partial support during the completion of this work.

[1] R. Maruyama et al., Physica (Amsterdam) 335B, 238 (2003); Nucl. Instrum. Methods Phys. Res., Sect. A 530, 505 (2004); H. Hayashida et al., Nucl. Instrum. Methods Phys. Res., Sect. A 574, 292 (2007).

[2] A. Abragam, Principles of Nuclear Magnetism (Oxford University Press, New York, 1996).

[3] Y. V. Taran, Dubna Report No. JINR P3-8577, Dubna, 1975.

[4] V. I. Luschikov and Y. V. Taran, Nucl. Instrum. Methods 228, 159 (1984).

[5] V. F. Ezhov et al., JETP Lett. 24, 34 (1976).

[6] A. N. Bazhenov, V. M. Lobashev, A. N. Pirozhkov, and V. N. Suslar, Nucl. Instrum. Methods Phys. Res., Sect. A 332, 534 (1993).

[7] S. V. Grigoriev, A. I. Okorokov, and V. V. Runov, Nucl. Instrum. Methods Phys. Res., Sect. A 384, 451 (1997).

[8] S. V. Grigoriev, R. Kreuger, W. H. Kraan, F. M. Mulder, and M. T. Rekveldt, Phys. Rev. A 64, 013614 (2001).

[9] J.D. Bowman et al., Report No. LA-UR-1999-5432, available at web site http://sns.phys.utk.edu/npdg/; W. M. Snow et al., Nucl. Instrum. Methods Phys. Res., Sect. A 440, 729 (2000); S. A. Page et al., J. Res. Natl. Inst. Stand. Technol. 110, 195 (2005).

[10] B. Desplanques, J. F. Donoghue, and B. R. Holstein, Ann. Phys. (N.Y.) 124, 449 (1980).

[11] R. Golub, R. Gahler, and T. Keller, Am. J. Phys. 62, 779 (1994).

[12] E. Kruger, Nukleonika 25, 889 (1980).

[13] G. J. Russell et al., Proceedings of the 16th Meeting of the International Conference on Advanced Neutron Sources, Düsseldorf-Neuss, Germany, 2003, p. 833. 
[14] P.-N. Seo et al., J. Res. Natl. Inst. Stand. Technol. 110, 145 (2005); Proceedings of the International Conference on Nuclear Data for Science and Technology (ND2004), edited by R. C. Haight, M. B. Chadwick, T. Kawano, and P. Talou, AIP Conference Proc. No. 769 (AIP, New York, 2005), p. 696.

[15] LND, Inc., 3230 Lawson Blvd. Oceanside, New York 11572; C. Gillis, Master's thesis, University of Manitoba, 2006 (unpublished).

[16] L. Barrón Palos et al. (unpublished).

[17] T.E. Chupp et al., Nucl. Instrum. Methods Phys. Res., Sect. A 574, 500 (2007).

[18] D. R. Rich et al., Nucl. Instrum. Methods Phys. Res., Sect. A 481, 431 (2002).
[19] M. Gericke et al., Nucl. Instrum. Methods Phys. Res., Sect. A 540, 328 (2005).

[20] W.S. Wilburn et al., Nucl. Instrum. Methods Phys. Res., Sect. A 540, 180 (2005).

[21] M. Gericke et al., Phys. Rev. C 74, 065503 (2006).

[22] M. Dabaghyan et al. (private communication).

[23] G. S. Mitchell et al., Nucl. Instrum. Methods Phys. Res., Sect. A 521, 468 (2004); V. A. Vesna et al., JETP Lett. 36, 209 (1982); M. Avenier et al., Nucl. Phys. A 436, 83 (1985).

[24] S. I. Penttila and J. D. Bowman, J. Res. Natl. Inst. Stand. Technol. 110, 309 (2005). 